\title{
Plug and Play Robust Distributed Control with Ellipsoidal Parametric Uncertainty System
}

\author{
Hong Wang-jian ${ }^{1}$ and Wang Yan-xiang ${ }^{2}$ \\ ${ }^{1}$ Dipartimento di Elettronica, Informazione, Politecnico di Milano, Piazza Leonardo da Vinci 32, 20133 Milano, Italy \\ ${ }^{2}$ School of Mechanical and Electronic Engineering, Jingdezhen Ceramic Institute, Jingdezhen 333403, China \\ Correspondence should be addressed to Wang Yan-xiang; wangyanxiang1@jci.edu.cn
}

Received 4 August 2016; Revised 10 October 2016; Accepted 31 October 2016

Academic Editor: Haibo Du

Copyright (c) $2016 \mathrm{H}$. Wang-jian and W. Yan-xiang. This is an open access article distributed under the Creative Commons Attribution License, which permits unrestricted use, distribution, and reproduction in any medium, provided the original work is properly cited.

\begin{abstract}
We consider a continuous linear time invariant system with ellipsoidal parametric uncertainty structured into subsystems. Since the design of a local controller uses only information on a subsystem and its neighbours, we combine the plug and play idea and robust distributed control to propose one distributed control strategy for linear system with ellipsoidal parametric uncertainty. Firstly for linear system with ellipsoidal parametric uncertainty, a necessary and sufficient condition for robust state feedback control is proposed by means of linear matrix inequality. If this necessary and sufficient condition is satisfied, this robust state feedback gain matrix can be easily derived to guarantee robust stability and prescribed closed loop performance. Secondly the plug and play idea is introduced in the design process. Finally by one example of aircraft flutter model parameter identification, the efficiency of the proposed control strategy can be easily realized.
\end{abstract}

\section{Introduction}

Modern engineering system offers many examples of manmade systems characterized by a large numbers of states and inputs. One concept about large-scale systems is always thought as the result of many subsystems interacting through the coupling of physical variables or the transmission of information over one communication network. Complexity of these large-scale systems brings some challenges such as the application of simulation, analysis, and control design algorithms.

Consider the problem of control design algorithms for large-scale systems; two common used algorithms are centralized control scheme and distributed control scheme. In centralized control scheme, all control variables are computed by a single regulator. Therefore all subsystems transmit their outputs to the central controller in order to design the control inputs which will be sent back to actuators collocated with subsystems. But a centralized control scheme for the large-scale systems will suffer from many problems such as (1) computational complexity, a centralized regulator needs a considerable amount of computing power and memory in order to compute control inputs within a sampling interval, (2) communication network, a centralized control requires a star-like topology of the communication network that impacts the cost of the control system, and (3) reliability, a failure in a single subsystem or in a link could comprise the proper functioning of the overall controlled large-scale system. In order to remedy the above drawbacks, each subsystem in distributed control scheme is equipped with a local controller that receives the outputs from the corresponding subsystem and computes the control inputs. It means that each subsystem is equipped with a local controller, but controllers can transmit and receive quantities from other subsystems. Therefore if these pieces of information are properly used, the goal of stabilizing the closed loop large-scale system and guaranteeing prescribed level of performance can be achieved.

Now one new idea of distributed control scheme is proposed to design the local controller in large-scale systems in reference [1], where the design of a local controller uses only information from parent subsystems. This new 
approach has several advantages. First, the communication flow at the design stage has the same topology of the coupling graph that is usually sparse. Second, after each parent subsystem has sent required quantities to its children, the design of local controllers can be finished in parallel. Third, the complexity of synthesizing a local controller for a subsystem scales only with the number of its parents rather than the total number of subsystems. Fourth, if a subsystem joins an existing network, at most, subsystems that are influenced by it can retune their controllers. In a similar way, if a subsystem leaves the network, at most, its children can retune their controllers. In [1], this kind of distributed control scheme is named as the plug and play distributed control. The main essence of plug and play distributed control scheme is that, when a subsystem joins or leaves an existing network of subsystems, there is a procedure for automatically assessing if the operation does not spoil stability and constraint satisfaction for the overall large-scale system. In these years, research on plug and play distributed control is being untaken. In [2], a linear system structured into physically coupled subsystem was considered, further one plug and play distributed model predictive control was proposed to guarantee asymptotic stability and satisfaction of constraints on system inputs and states. Reference [3] considered the problem of designing distributed controllers for large-scale linear constrained systems composed by a number of interacting subsystems. Several aspects of plug and play distributed control were improved in [4], and based on the computation of robust control invariant sets, all critical steps in the design of a local controller could be solved through linear programming strategy. Reference [5] considered the control of a large-scale system composed of state coupled linear subsystems that could be added or removed offline; the possibility of coping with constraints on system variables was studied in [6], while guaranteeing stability, robustness, and global optimality. Plug and play distributed methods span from cooperative to noncooperative, which requires limited computational load in [7]. A review about architectures for distributed model predictive control can be seen in [8]. Generally plug and play distributed control procedures are very attractive for large-scale systems where the number of subsystems varies with time [9]. And the plug and play idea provides a scalable procedure for the addition and removal of new generation units [10]. Furthermore, plug and play distributed controllers can facilitate the revamping of control systems and allow one to automatically assess feasibility of the whole process.

Using above descriptions and advantages of plug and play distributed control, in this short note, we apply this new plug and play idea into the robust control scheme to propose one new method (plug and play robust distributed control method). The goal of the robust control scheme is to guarantee that one $H \infty$ norm with respect to one transfer function from external noise to system output is less than one given upper bound [11]. Here the controller is the common stable state feedback controller, and under the condition that the assumed model structure is given previously, we solve the problem of how to design one robust distributed controller with ellipsoidal parametric uncertainty system. First, we derive one necessary and sufficient condition under which the robust distributed controller exists by using the linear matrix inequality tool for each subsystem. This necessary and sufficient condition of linear matrix inequality form can guarantee the robust stability and performance requirement. Then the distributed state feedback controller is chosen as one matrix from the state space matrix form directly [12]. Second, the idea of plug and play is merged into the robust distributed control and one plug and play robust distributed control algorithm is formulated. Finally, some simulation examples are used to illustrate the efficiency of our new method.

\section{Model Description}

Consider a continuous time linear invariant system.

$$
\begin{aligned}
& \dot{x}(t)=A x(t)+B_{1} u(t), \\
& y(t)=C(\theta) x(t)+B_{2} u(t),
\end{aligned}
$$

where $x(t) \in R^{n}$ and $u(t) \in R^{m}$ are state and input, respectively, at time $t$ and $y(t) \in R^{p}$ is output. Then the above four matrices satisfy

$$
\begin{array}{r}
A \in R^{n \times n}, \\
B_{1} \in R^{n \times m}, \\
C(\theta) \in R^{p \times n}, \\
B_{2} \in R^{p \times m} .
\end{array}
$$

As one unknown parameter vector $\theta \in R^{n}$ lies in matrix $C(\theta)$, (1) is a parameterized model. Assume all matrices are known a priori except for matrix $C(\theta)$, this assumption signifies that (1) has uncertain zeros [13]. Assume that the unknown parameter vector $\theta$ is in one given ellipsoidal parametric set $U_{\theta}$.

$$
U_{\theta}=\left\{\theta:\left(\theta-\theta_{0}\right)^{T} R\left(\theta-\theta_{0}\right) \leq 1\right\},
$$

where variables $\theta_{0}$ and $R$ are used to express the center and volume corresponding to the above given ellipsoidal parametric set $U_{\theta}$. The state $x(t)=\left(\begin{array}{llll}x_{1}(t) & x_{2}(t) & \cdots & x_{M}(t)\end{array}\right)$ is partitioned into $M$ vector $x_{i}(t) \in R^{n_{i}}$, where $i \in M=1: M$ and $n=\sum_{i \in M} n_{i}$. Similarly the input and the output are composed by $M$ vector $u_{i}(t) \in R^{m_{i}}, y_{i}(t) \in R^{p_{i}}$ such that

$$
\begin{aligned}
& u(t)=\left(\begin{array}{lllll}
u_{1}(t) & u_{2}(t) & \cdots & \left.u_{M}(t)\right), \quad m=\sum_{i \in M} m_{i}
\end{array}\right. \\
& y(t)=\left(\begin{array}{llll}
y_{1}(t) & y_{2}(t) & \cdots & y_{M}(t)
\end{array}\right), \quad p=\sum_{i \in M} p_{i} .
\end{aligned}
$$

Assume that (1) can be equivalently described by the $i$ th subsystem $\left(\Sigma_{i}\right), i \in M$, given by

$\left(\Sigma_{i}\right)$

$$
\begin{aligned}
& \dot{x}_{i}(t)=A_{i i} x_{i}(t)+B_{1 i} u_{i}(t)+\sum_{i \in N_{i}} A_{i j} x_{j}(t) \\
& y_{i}(t)=C_{i}\left(\theta_{i}\right) x_{i}(t)+B_{2 i} u_{i}(t),
\end{aligned}
$$


where

$$
\begin{aligned}
A_{i i} & \in R^{n_{i} \times n_{i}}, \\
B_{1 i} & \in R^{n_{i} \times m_{i}}, \\
C_{i}\left(\theta_{i}\right) & \in R^{p_{i} \times n_{i}}, \\
B_{2 i} & \in R^{p_{i} \times m_{i}}, \\
A_{i j} & \in R^{n_{i} \times n_{j}}, \\
& \quad i, j \in M .
\end{aligned}
$$

$N_{i}$ is the set of parents of subsystem $i$, and it is defined as

$$
N_{i}=\left\{j \in M: A_{i j} \neq 0, i \neq j\right\} .
$$

Further since $y_{i}(t)$ depends on the local state $x_{i}(t)$ only, subsystems $\left(\Sigma_{i}\right), i \in M$ are output-decoupled and $C=$ $\operatorname{diag}\left(\begin{array}{lllll}C_{1} & C_{2} & \cdots & C_{M}\end{array}\right)$. Also the unknown parameter vector $\theta \in R^{n}$ is partitioned into the $M$ vector $\theta_{i} \in R^{n_{i}}$, where $i \in M=1: M$ and $n=\sum_{i \in M} n_{i}$.

We treat $w_{i}(t)=\sum_{i \in N_{i}} A_{i j} x_{j}(t)$ as a disturbance and reformulate (5) as follows:

$$
\begin{aligned}
& \left(\Sigma_{i}\right) \\
& \dot{x}_{i}(t)=A_{i i} x_{i}(t)+B_{1 i} u_{i}(t)+B_{3 i} w_{i}(t) \\
& y_{i}(t)=C_{i}\left(\theta_{i}\right) x_{i}(t)+B_{2 i} u_{i}(t),
\end{aligned}
$$

where $w_{i}(t) \in R^{n_{i}}, B_{3 i} \in R^{n_{i} \times n_{i}}$ and vector $\theta_{i} \in R^{n_{i}}$ is also in one given ellipsoidal parametric set $U_{\theta_{i}}$.

$$
U_{\theta_{i}}=\left\{\theta_{i}:\left(\theta_{i}-\theta_{i 0}\right)^{T} R_{i}\left(\theta_{i}-\theta_{i 0}\right) \leq 1\right\},
$$

where variables $\theta_{i 0}$ and $R_{i}$ are all similar to above definitions. After combining (8) and (9), the problem of our paper is to design one stable distributed state feedback controller $u_{i}(t)=K_{i} x_{i}(t)$ under the condition of disturbance $w_{i}(t)$ and ellipsoidal parametric set $U_{\theta_{i}}$. The state feedback matrix $K_{i}$ satisfies that $K_{i} \in R^{m_{i} \times n_{i}}$.

For the convergence of next analysis, we need the following two assumptions to express that subsystems $\left(\Sigma_{i}\right), i \in M$ are state coupled and input decoupled.

Assumption 1. Matrix $A$ is composed by blocks $A_{i j}, \forall i, j \in M$ and $B=\operatorname{diag}\left(\begin{array}{llll}B_{1} & B_{2} & \cdots & B_{M}\end{array}\right)$.

The physical meaning of assumption 1 is that each subsystem is coupled through state variables only. In reference [14], this type of coupling is sometimes called dynamic coupling. This dynamic coupling exists in many communication networks.

Assumption 2. The matrix pairs $\left(A_{i i}, B_{i}\right), \forall i \in M$ are controllable.

Under these two assumptions, if one stable distributed state feedback controller $u_{i}(t)=K_{i} x_{i}(t)$ is obtained, then the controller

$$
\begin{aligned}
u(t) & =\left(\begin{array}{llll}
u_{1}(t) & u_{2}(t) & \cdots & u_{M}(t)
\end{array}\right) \\
& =\left(\begin{array}{lllll}
K_{1} x_{1}(t) & K_{2} x_{2}(t) & \cdots & K_{M} x_{M}(t)
\end{array}\right)
\end{aligned}
$$

can be used to control system (1), while guaranteeing stability and robustness [15]. By observing (8) again, we obtain one closed loop transfer function $T_{i}\left(q, \theta_{i}\right)$ from external disturbance to system output $y_{i}(t)$.

$$
T_{i}\left(q, \theta_{i}\right)=\left(C_{i}+B_{2 i} K_{i}\right)\left(q I-A_{i i}-B_{1 i} K_{i}\right)^{-1} B_{3 i} .
$$

The goal of robust distributed control is to find one stable feedback controller $K_{i}$ to satisfy the following inequality.

$$
\left\|T_{i}\left(q, \theta_{i}\right)\right\|_{\infty}<\gamma, \quad \forall \theta_{i} \in U_{\theta_{i}},
$$

where $\gamma$ is a given upper bound, and $H \infty$ norm is defined as.

$$
\begin{aligned}
\left\|T_{i}\left(q, \theta_{i}\right)\right\|_{\infty} & =\sup _{w \in[0,2 \pi]}\left\|T_{i}\left(e^{j w}, \theta_{i}\right)\right\| \\
& =\sup _{w \in[0,2 \pi]} \sigma\left[T_{i}\left(e^{j w}, \theta_{i}\right)\right],
\end{aligned}
$$

where $\|\cdot\|$ denotes the maximal singular value; the existence of that stable state feedback controller $K_{i}$ satisfying inequality (12) can be verified by a linear matrix inequality condition [16]. Further this linear matrix inequality condition is a necessary and sufficient condition in the robust control theory.

\section{One Necessary and Sufficient Condition}

In this section, we derive one linear matrix inequality condition in one state feedback $H \infty$ problem. Further in this problem, the unknown parameter vector $\theta_{i}$ is known in one given ellipsoidal parametric set $U_{\theta_{i}}$. This linear matrix inequality condition can be formulated as the following Theorem 3 .

Theorem 3. Assume the following equality holds

$$
B_{2 i}^{T}\left[\begin{array}{ll}
C_{i} & B_{2 i}
\end{array}\right]=\left[\begin{array}{ll}
0 & I
\end{array}\right] .
$$

Then the following two statements are equivalent to each other:

(1) There exists one state feedback controller

$$
u_{i}(t)=K_{i} x_{i}(t)
$$

which satisfies $\left\|T_{i}\left(q, \theta_{i}\right)\right\|_{\infty}<\gamma$.

(2) There exists a symmetric matrix $X_{i} \in R^{n_{i} \times n_{i}}$ such that

$$
\begin{aligned}
& {\left[\begin{array}{cc}
A_{i i} X_{i}+X_{i} A_{i i}^{T}-\gamma^{2}\left(B_{1 i} B_{1 i}^{T}-B_{3 i} B_{3 i}^{T}\right) & X_{i} C_{i}^{T}\left(\theta_{i}\right) \\
C_{i}\left(\theta_{i}\right) X_{i} & -I
\end{array}\right]} \\
& \quad<0 .
\end{aligned}
$$

Proof. From the results in [17], we see that the L2 gain of one linear time invariant system is equivalent to the $H \infty$ norm of its transfer matrix function. Then the condition that $H \infty$ norm of one transfer matrix function is less than scalar $\gamma$ can be transformed to that its $L 2$ gain is less than scalar $\gamma$. Furthermore the necessary and sufficient condition that the $L 2$ gain is less than scalar $\gamma$ can be reformulated as follows. 
Taking one quadratic function as that,

$$
V_{i}\left(x_{i}(t)\right)=x_{i}^{T}(t) P_{i} x_{i}(t), \quad P_{i}>0,
$$

such that, for all variables $t$, we have

$$
\frac{d V_{i}\left(x_{i}(t)\right)}{d t}+y_{i}^{T}(t) y_{i}(t)-\gamma^{2} w_{i}^{T}(t) w_{i}(t)<0
$$

Substituting $u_{i}(t)=K_{i} x_{i}(t)$ into (8), we obtain.
$\left(\Sigma_{i}\right)$

$$
\begin{aligned}
\dot{x}_{i}(t) & =\left[A_{i i}+B_{1 i} K_{i}\right] x_{i}(t)+B_{3 i} w_{i}(t), \\
y_{i}(t) & =\left[C_{i}\left(\theta_{i}\right)+B_{2 i} K_{i}\right] x_{i}(t) .
\end{aligned}
$$

Substituting equation (19) into (18), we see that

$$
\begin{gathered}
\dot{x}_{i}^{T}(t) P_{i} x_{i}(t)+x_{i}^{T}(t) P_{i} \dot{x}_{i}^{T}(t)+y_{i}^{T}(t) y_{i}(t) \\
-\gamma^{2} w_{i}^{T}(t) w_{i}(t)<0 .
\end{gathered}
$$

Through complex mathematical operations, one linear matrix inequality is obtained.

$$
\left[\begin{array}{cr}
{\left[A_{i i}+B_{1 i} K_{i}\right]^{T} P_{i}+P_{i}\left[A_{i i}+B_{1 i} K_{i}\right]+\left[C_{i}\left(\theta_{i}\right)+B_{2 i} K_{i}\right]^{T}\left[C_{i}\left(\theta_{i}\right)+B_{2 i} K_{i}\right]} & P_{i} B_{3 i} \\
B_{3 i}^{T} P_{i} & -\gamma^{2} I
\end{array}\right]<0 .
$$

Equation (21) is equivalent to the notion that there exists $K_{i}$ and $Q_{i}=P_{i}^{-1}$ such that

$$
\left[\begin{array}{cc}
{\left[A_{i i}+B_{1 i} K_{i}\right]^{T} Q_{i}+Q_{i}\left[A_{i i}+B_{1 i} K_{i}\right]+B_{3 i} B_{3 i}^{T}} & Q_{i}\left[C_{i}\left(\theta_{i}\right)+B_{2 i} K_{i}\right]^{T} \\
{\left[C_{i}\left(\theta_{i}\right)+B_{2 i} K_{i}\right] Q_{i}} & -\gamma^{2} I
\end{array}\right]<0 .
$$

Introduce one new variable $Y_{i}=K_{i} Q_{i}$, and then (22) can be rewritten as

$$
\left[\begin{array}{cc}
A_{i i} Q_{i}+Q_{i} A_{i i}^{T}+Y_{i}^{T} B_{1 i}+B_{1 i} Y_{i}+B_{3 i} B_{3 i}^{T} & {\left[C_{i}\left(\theta_{i}\right) Q_{i}+B_{2 i} Y_{i}\right]^{T}} \\
{\left[C_{i}\left(\theta_{i}\right) Q_{i}+B_{2 i} Y_{i}\right]} & -\gamma^{2} I
\end{array}\right]<0
$$

Applying the Schur complement formula [18], (23) is equivalent to the following inequality:

$$
\begin{aligned}
A_{i i} Q_{i} & +Q_{i} A_{i i}^{T}+Y_{i}^{T} B_{1 i}+B_{1 i} Y_{i}+B_{3 i} B_{3 i}^{T} \\
& +\frac{\left[C_{i}\left(\theta_{i}\right) Q_{i}+B_{2 i} Y_{i}\right]^{T}\left[C_{i}\left(\theta_{i}\right) Q_{i}+B_{2 i} Y_{i}\right]}{\gamma^{2}}<0 .
\end{aligned}
$$

If (14) holds, then (24) can be simplified as

$$
\begin{aligned}
A_{i i} Q_{i}+Q_{i} A_{i i}^{T}+Y_{i}^{T} B_{1 i}+B_{1 i} Y_{i}+B_{3 i} B_{3 i}^{T} \\
+\frac{Q_{i} C_{i}^{T}\left(\theta_{i}\right) C_{i}\left(\theta_{i}\right) Q_{i}+Y_{i}^{T} Y_{i}}{\gamma^{2}}<0 .
\end{aligned}
$$

Equation (25) is equivalent to the following inequality:

$$
\begin{array}{r}
A_{i i} Q_{i}+Q_{i} A_{i i}^{T}-B_{1 i} B_{1 i}^{T}+B_{3 i} B_{3 i}^{T} \\
+\frac{Q_{i} C_{i}^{T}\left(\theta_{i}\right) C_{i}\left(\theta_{i}\right) Q_{i}}{\gamma^{2}}<0 .
\end{array}
$$

Applying the Schur complement formula again, we obtain.

$$
\begin{aligned}
& {\left[\begin{array}{cc}
A_{i i} X_{i}+X_{i} A_{i i}^{T}-\gamma^{2}\left(B_{1 i} B_{1 i}^{T}-B_{3 i} B_{3 i}^{T}\right) & X_{i} C_{i}^{T}\left(\theta_{i}\right) \\
C_{i}\left(\theta_{i}\right) X_{i} & -I
\end{array}\right]} \\
& \quad<0,
\end{aligned}
$$

where we use a new variable $X_{i}=\gamma^{2} Q_{i}$, thus concluding the proof.

Theorem 3 gives one necessary and sufficient condition for the existence of a robust controller when the parameter vector is uncertain.

Now by using that linear matrix inequality (16), we continue to study one special case; that is, matrix $C_{i}\left(\theta_{i}\right)$ is written as the following special structure:

$$
C_{i}\left(\theta_{i}\right)=\left(\begin{array}{ll}
\theta_{i} & 0
\end{array}\right)^{T} \in R^{n_{i} \times n_{i}} .
$$

Then the necessary and sufficient condition with respect to this special case can be reformulated as Theorem 4 . 
Theorem 4. Consider the parameterized system structure (8), and matrix $C_{i}\left(\theta_{i}\right)$ is given as (28) and other matrices are known. Assume (14) also holds, and then the following two statements are equivalent:

(1) There exists one state feedback controller

$$
u_{i}(t)=K_{i} x_{i}(t)
$$

which satisfies $\left\|T_{i}\left(q, \theta_{i}\right)\right\|_{\infty}<\gamma$ for all $\theta_{i} \in U_{\theta_{i}}$.

(2) There exists a symmetric matrix $X_{i} \in R^{n_{i} \times n_{i}}$ and one scalar $\tau \in R, \tau>0$ such that

$$
\begin{aligned}
& {\left[\begin{array}{ccc}
\tau R_{i} & X_{i} & \tau R_{i} \theta_{i 0} \\
X_{i} & -M_{i} & 0 \\
\tau \theta_{i 0}^{T} R_{i} & 0 & 1+\left(\tau \theta_{i 0}^{T} R_{i} \theta_{i 0}-1\right)
\end{array}\right]>0,} \\
& M_{i}=A_{i i} X_{i}+X_{i} A_{i i}^{T}-\gamma^{2}\left(B_{1 i} B_{1 i}^{T}-B_{3 i} B_{3 i}^{T}\right) .
\end{aligned}
$$

Proof. Considering system (8) and (28), we continue to compute that

$$
C_{i}\left(\theta_{i}\right) X_{i}=\left(\begin{array}{c}
\theta_{i}^{T} \\
0
\end{array}\right) X_{i}=\left(\begin{array}{c}
\theta_{i}^{T} X_{i} \\
0
\end{array}\right)
$$

Substituting (31) into (16), then that linear matrix inequality is rewritten as

$$
\left[\begin{array}{ccc}
A_{i i} X_{i}+X_{i} A_{i i}^{T}-\gamma^{2}\left(B_{1 i} B_{1 i}^{T}-B_{3 i} B_{3 i}^{T}\right) & X_{i} \theta_{i} & 0 \\
\theta_{i}^{T} X_{i} & -I & 0 \\
0 & 0 & 0
\end{array}\right]<0 .
$$

According to the structure of $X_{i} \theta_{i}$, we simplify the above (32) as

$$
\left[\begin{array}{cc}
M_{i} & X_{i} \theta_{i} \\
\theta_{i}^{T} X_{i} & -I
\end{array}\right]<0
$$

From Schur complement formula, we see that linear matrix inequality (33) is equivalent to the following two linear matrix inequalities:

$$
\begin{aligned}
M_{i} & <0, \\
-1-\theta_{i}^{T} X_{i} M_{i}^{-1} X_{i} \theta_{i} & <0 .
\end{aligned}
$$

Rewrite (34) as

$$
\left(\begin{array}{c}
\theta_{i} \\
1
\end{array}\right)^{T}\left(\begin{array}{cc}
-X_{i} M_{i}^{-1} X_{i} & 0 \\
0 & -1
\end{array}\right)\left(\begin{array}{c}
\theta_{i} \\
1
\end{array}\right)<0 .
$$

Similarly the ellipsoidal parameter set $U_{\theta_{i}}$ is rewritten as

$$
\left(\begin{array}{c}
\theta_{i} \\
1
\end{array}\right)^{T}\left(\begin{array}{cc}
R_{i} & -R_{i} \theta_{i 0} \\
-\theta_{i 0}^{T} R_{i} & \theta_{i 0}^{T} R_{i} \theta_{i 0}-1
\end{array}\right)\left(\begin{array}{c}
\theta_{i} \\
1
\end{array}\right)<0
$$

Applying S-Procedure strategy [18] and combining (35) and (36), there exists one scalar $\tau>0$, such that

$$
\left(\begin{array}{cc}
-X_{i} M_{i}^{-1} X_{i} & 0 \\
0 & -1
\end{array}\right)-\tau\left(\begin{array}{cc}
R_{i} & -R_{i} \theta_{i 0} \\
-\theta_{i 0}^{T} R_{i} & \theta_{i 0}^{T} R_{i} \theta_{i 0}-1
\end{array}\right)<0 .
$$

Rewriting (37), we obtain one simplified form.

$$
\left(\begin{array}{cc}
-X_{i} M_{i}^{-1} X_{i}-\tau R_{i} & \tau R_{i} \theta_{i 0} \\
\tau \theta_{i 0}^{T} R_{i} & -1-\tau\left(\theta_{i 0}^{T} R_{i} \theta_{i 0}-1\right)
\end{array}\right)<0 .
$$

Applying the Schur complement formula, linear matrix inequality (38) is equivalent to the following two linear matrix inequalities:

$$
\begin{array}{r}
-1-\tau\left(\theta_{i 0}^{T} R_{i} \theta_{i 0}-1\right)<0, \\
-X_{i} M_{i}^{-1} X_{i}-\tau R_{i}-\frac{\tau^{2} R_{i} \theta_{i 0} \theta_{i 0}^{T} R_{i}}{-1-\tau\left(\theta_{i 0}^{T} R_{i} \theta_{i 0}-1\right)}<0 .
\end{array}
$$

It means that

$$
\begin{array}{r}
1+\tau\left(\theta_{i 0}^{T} R_{i} \theta_{i 0}-1\right)>0, \\
X_{i} M_{i}^{-1} X_{i}+\tau R_{i}-\frac{\tau^{2} R_{i} \theta_{i 0} \theta_{i 0}^{T} R_{i}}{1+\tau\left(\theta_{i 0}^{T} R_{i} \theta_{i 0}-1\right)}>0 .
\end{array}
$$

Combining (34) and (40) and then using Schur complement formula, we get

$$
\left[\begin{array}{cc}
\tau R_{i}-\frac{\tau^{2} R_{i} \theta_{i 0} \theta_{i 0}^{T} R_{i}}{1+\tau\left(\theta_{i 0}^{T} R_{i} \theta_{i 0}-1\right)} & X_{i} \\
X_{i} & -M_{i}
\end{array}\right]>0 .
$$

Because linear matrix inequality (41) is linear with respect to variable $X_{i}$ and nonlinear of variable $\tau$, rewrite (41) as

$$
\begin{aligned}
& {\left[\begin{array}{cc}
\tau R_{i} & X_{i} \\
X_{i} & -M_{i}
\end{array}\right]} \\
& \quad-\left[\begin{array}{c}
\tau R_{i} \theta_{i 0} \\
0
\end{array}\right] \frac{1}{1+\tau\left(\theta_{i 0}^{T} R_{i} \theta_{i 0}-1\right)}\left[\begin{array}{c}
\tau R_{i} \theta_{i 0} \\
0
\end{array}\right]^{T}>0 .
\end{aligned}
$$

Applying Schur complement formula again, the result (32) can be obtained, thus concluding the proof.

As linear matrix inequality (30) is linear with respect to variables $X_{i}, \tau$, and $\gamma^{2}$, then that minimization performance bound will give a necessary and sufficient condition for the existence of the robust distributed controller. From the robust control theory, we conclude that when the above necessary and sufficient condition is satisfied, then one robust distributed control state feedback controller $K_{i}$ can be chosen as the following form directly:

$$
K_{i}=-\left(B_{2 i}^{T} B_{2 i}\right) B_{1 i}^{T}=-B_{1 i}^{T},
$$

where the second equality holds under the condition of (14). 


\section{Plug and Play Idea}

In this section, we study the plug and play idea and the redesign of new controllers when subsystems are added to or removed from system (8). Note that plugging in and unplugging of subsystems are regarded as offline operation [19].

(1) As a starting point, consider a system composed by subsystems $\left(\sum_{i}\right), i \in M$ with its robust distributed controller $u_{i}, i \in M$.

Consider the plugging of subsystem $\left(\Sigma_{M+1}\right)$, which is characterized by variables as follows:

$$
\begin{aligned}
& A_{M+1, M+1}, B_{1, M+1}, B_{2, M+1}, B_{3, M+1}, C_{M+1}, x_{M+1}, u_{M+1}, \\
& \quad N_{M+1},\left(A_{i j}\right)_{j \in N_{M+1}}
\end{aligned}
$$

into an existing system structure. In order to design controller $u_{M+1}$, we verify whether (16) in Theorem 3 holds; if it holds, then the robust distributed state feedback controller $u_{M+1}$ can be chosen as form (43). If the above design process stops, we deem that subsystem $\left(\Sigma_{M+1}\right)$ cannot plug in. Set $S_{i}=\{j$ : $\left.i \in N_{j}\right\}$ to be the set of successors to system $i$, since each subsystem $\left(\Sigma_{j}\right), j \in S_{M+1}$ has the new neighbour $\left(\Sigma_{M+1}\right)$. When $N_{j}$ gets larger, the necessary and sufficient condition will be violated. It means that, for each $j \in S_{M+1}$, the controller $u_{j}$ must be redesigned. Furthermore the addition of system $\left(\Sigma_{M+1}\right)$ triggers the design of controller $u_{M+1}$ and the redesign of controllers $u_{j}, j \in S_{M+1}$. And the controller redesign does not propagate further in the network system.

(2) When subsystem $\left(\Sigma_{k}\right), k \in M$ is removed off from the existing system, since, for each $i \in S_{k}$, the set $N_{i}$ gets smaller. The size of the ellipsoidal parametric uncertainty set cannot increase and therefore the linear matrix inequality cannot be violated. It means that, for each $i \in S_{k}$, the controller $u_{i}$ does not have to be redesigned. Further since, for each system $\left(\Sigma_{j}\right)$, $j \notin\{k\} \cup S_{k}$, the set $N_{j}$ does not change, the redesign of controller $u_{i}$ is not required.

(3) Generally after combing all above descriptions, one plug and play robust distributed control algorithm is formulated as follows.

Plug and Play Algorithm. Design of controller $u(t)$ for system (1).

Input. $A, B_{1}, B_{2}, C(\theta), x(t), y(t), u_{\theta}$

Output. $u(t)$

(1) Partition the original continuous time linear invariant system as (8) to obtain $M$ subsystems

$$
\sum_{i}, \quad i \in M
$$

(2) Compute matrices and other variables such as $A_{i i}, B_{1 i}$, $B_{2 i}, B_{3 i}, C_{i}\left(\theta_{i}\right), x_{i}(t), y_{i}(t), u_{\theta_{i}}$.

(3) Use linear matrix inequality tools from MATLAB 2009 to find one symmetric matrix $X_{i}$ such that inequality (16) holds.
(4) Find one appropriate state feedback controller $u_{i}(t)$ as in (43).

(5) Merge the plug and play idea during the above process to verify all $M$ inequalities (16).

(6) Construct the original control input as

$$
u(t)=\left(\begin{array}{llll}
u_{1}(t) & u_{2}(t) & \cdots & u_{M}(t)
\end{array}\right)
$$

\section{Simulation}

In this simulation part, we give one aircraft flutter model parameter identification example to confirm the efficiency of our plug and play robust distributed control strategy.

In the simulation example, we use one flutter mathematical model from [20]. In simulation environment, the input signal is the excited signal chosen by user, output is measured from the point set collected by the accelerometer, the number of sampled points is set $N=4096$, and sample instant is 1 second. The output and input of the 4096 sampled data are divided into 4 equal data blocks; each data block contains 1024 sample data. The true model matrices are listed as follows.

$$
\begin{aligned}
A & =\left[\begin{array}{ccc}
0 & 1 & 0 \\
-0.3 & 0.4 & -0.2 \\
-0.1 & 0.2 & 0.4
\end{array}\right], \\
B_{1} & =\left[\begin{array}{lll}
0.8 & 0.17 & 1.09
\end{array}\right]^{T}, \\
C & =\left[\begin{array}{lll}
2 & 1 & 0 \\
0 & 0 & 0
\end{array}\right], \\
B_{2} & =\left[\begin{array}{l}
0 \\
1
\end{array}\right], \\
\theta_{0} & =\left[\begin{array}{c}
0.5 \\
0.5 \\
-0.5
\end{array}\right] \\
R & =I \\
\gamma & =2 \\
\phi_{w} & =1
\end{aligned}
$$

$U_{\theta}$ is one guaranteed ellipsoidal parameter uncertain set with probability $95 \%$. To validate the identification result of matrix $C$, Figure 1 shows the approximation degree between the true system and the identified system. As those two curves approximate to each other very closely, the problem of one $H \infty$ norm with respect to one transfer function less than one given upper bound is equivalent to the problem of designing one robust state feedback controller. From Theorem 3, we see that the above problem is also equivalent to the following 

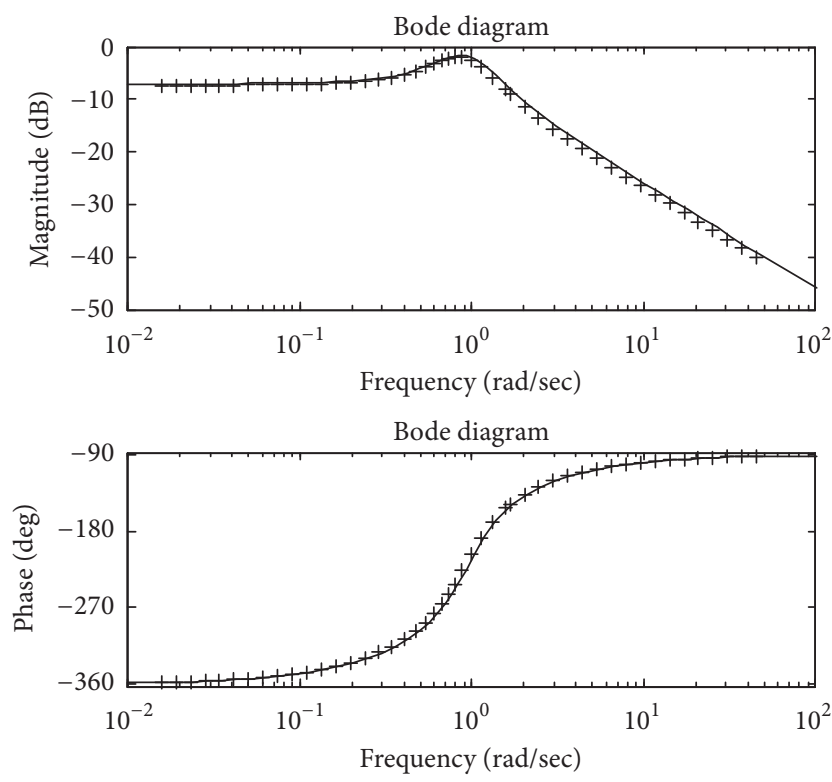

FIgURE 1: Bode plots compared between the true system and the identified system.

feasible problem with respect to linear matrix inequality. Find one symmetric matrix $X_{i} \in R^{n_{i} \times n_{i}}, \tau \in R, \tau>0$ such that

$$
\begin{aligned}
& {\left[\begin{array}{ccc}
\tau R_{i} & X_{i} & \tau R_{i} \theta_{i 0} \\
X_{i} & -M_{i} & 0 \\
\tau \theta_{i 0}^{T} R_{i} & 0 & 1+\left(\tau \theta_{i 0}^{T} R_{i} \theta_{i 0}-1\right)
\end{array}\right]>0,} \\
& M_{i}=A_{i i} X_{i}+X_{i} A_{i i}^{T}-\gamma^{2}\left(B_{1 i} B_{1 i}^{T}-B_{3 i} B_{3 i}^{T}\right) .
\end{aligned}
$$

The above linear matrix inequality can be solved by linear matrix inequality tools from MATLAB 2009; then this robust distributed sate feedback control $K_{i}=-\left(B_{2 i}^{T} B_{2 i}\right) B_{1 i}^{T}=-B_{1 i}^{T}$ can achieve the robust stability and performance requirement. To reduce the computational complexity, the plug and play idea is applied in the whole design process.

\section{Conclusion}

In this short paper, we propose one plug and play robust distributed state feedback control for one kind of continuous linear invariant systems with ellipsoidal parametric uncertain set. After partitioning the original system into some subsystems, we derive one necessary and sufficient condition under which the robust distributed controller exists by using the linear matrix inequality tool for each subsystem. Then we apply the new plug and play idea into the robust control scheme to propose one method-plug and play robust distributed control method. But all the derivations are based on the assumption that the unknown parameter is known in one ellipsoidal set. So when any knowledge of unknown parameter in the state space matrix form is not known a priori, we should apply the input-output data to design one system identification experiment and obtain its parameter estimation. Then the next topic is how to apply the system identification concept into our control strategy.

\section{Competing Interests}

The authors declare that there are no competing interests regarding the publication of this paper.

\section{References}

[1] S. Riverso, M. Farina, and G. Ferrari-Trecate, "Plug-and-play decentralized model predictive control for linear systems," IEEE Transactions on Automatic Control, vol. 58, no. 10, pp. 26082614, 2013.

[2] S. Riverso, M. Farina, and G. Ferrari-Trecate, "Plug-and-play model predictive control based on robust control invariant sets," Automatica, vol. 50, no. 8, pp. 2179-2186, 2014.

[3] S. Riverso and G. Ferrari-Trecate, "Tube-based distributed control of linear constrained systems," Automatica, vol. 48, no. 11, pp. 2860-2865, 2012.

[4] S. Riverso and G. Ferrari-Trecate, "Plug-and-play distributed model predictive control with coupling attenuation," Optimal Control Applications and Methods, vol. 36, no. 3, pp. 292-305, 2015.

[5] J. Lavaei and A. G. Aghdam, "Control of continuous time LTI systems by means of structurally constrained controllers," Automatica, vol. 44, no. 1, pp. 141-148, 2008.

[6] J. K. Scott, R. Findeisen, R. D. Braatz, and D. M. Raimondo, "Input design for guaranteed fault diagnosis using zonotopes," Automatica, vol. 50, no. 6, pp. 1580-1589, 2014.

[7] P. Trodden and A. Richards, "Distributed model predictive control of linear systems with persistent disturbances," International Journal of Control, vol. 83, no. 8, pp. 1653-1663, 2010.

[8] R. Scattolini, "Architectures for distributed and hierarchical Model Predictive Control-a review," Journal of Process Control, vol. 19, no. 5, pp. 723-731, 2009.

[9] A. Ferramosca, D. Limon, I. Alvarado, and E. F. Camacho, "Cooperative distributed MPC for tracking," Automatica, vol. 49, no. 4, pp. 906-914, 2013.

[10] D. M. Raimondo, L. Magni, and R. Scattolini, "Decentralized MPC of nonlinear systems: an input-to-state stability approach," International Journal of Robust and Nonlinear Control, vol. 17, no. 5, pp. 1651-1667, 2007.

[11] M. C. Campi and P. R. Kumar, "Learning dynamical systems in a stationary environment," Systems \& Control Letters, vol. 34, no. 3, pp. 125-132, 1998.

[12] S. Silven, "A neural network based optimization algorithm for multiple targets tracking," IEEE Journal of Oceanic Engineering, vol. 37, no. 4, pp. 56-62, 2010.

[13] F. Giulietti, "Dynamics and control issues of formation flight," Aerospace Science and Technology, vol. 36, no. 9, pp. 65-71, 2005.

[14] Y. Ben-Asher, S. Feldman, P. Gurfil, and M. Feldman, "Distributed decision and control for cooperative UAVs using ad hoc communication," IEEE Transactions on Control Systems Technology, vol. 16, no. 3, pp. 511-516, 2008.

[15] W.-H. Chen, "Nonlinear disturbance observer-enhanced dynamic inversion control of missiles," Journal of Guidance, Control, and Dynamics, vol. 26, no. 1, pp. 161-166, 2003.

[16] F. Giulietti, "Dynamics and control of different aircraft formation structures," Aeronautical, vol. 108, no. 10, pp. 117-124, 2004.

[17] J. H. Wang, "Fast gradient algorithm in subspace predictive control under fault estimation," Journal of Shanghai Jiao Tong University, vol. 47, no. 7, pp. 1015-1021, 2013. 
[18] Q. Lan, S. Ding, S. Li, and C. Zhang, "Global decentralised stabilisation for a class of uncertain large-scale feedforward nonlinear systems," International Journal of Control, vol. 87, no. 6, pp. 1282-1296, 2014.

[19] Q. Lan, C. Zhang, and S. Li, "Global decentralized stabilization for a class of large-scale feedforward nonlinear systems," in Proceedings of the American Control Conference (ACC '15), pp. 645650, July 2015.

[20] A. Dankers, P. M. J. Van den Hof, X. Bombois, and P. S. Heuberger, "Identification of dynamic models in complex networks with prediction error methods: predictor input selection," IEEE Transactions of Automatic Control, vol. 61, no. 4, pp. 937-952, 2016. 


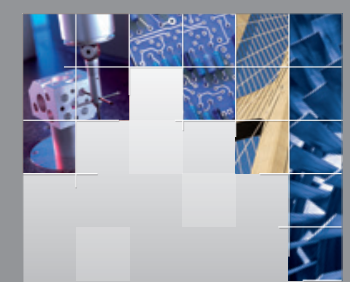

\section{Enfincering}
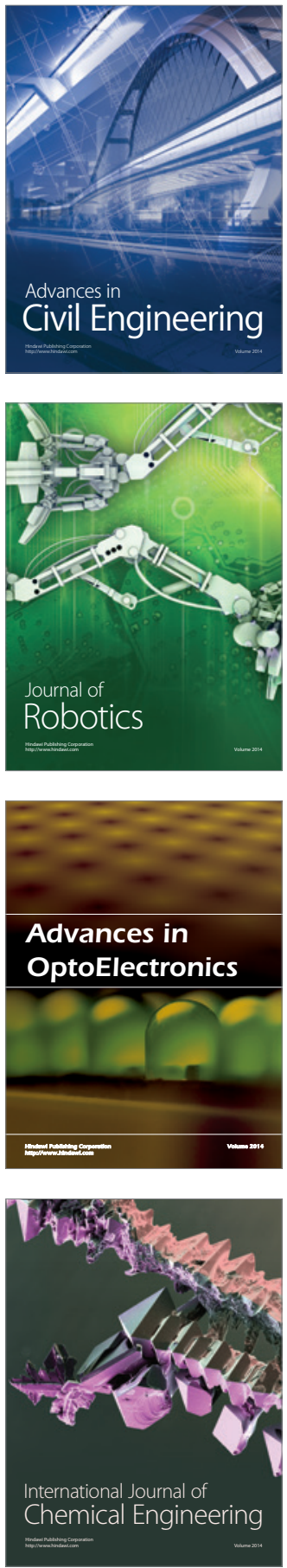

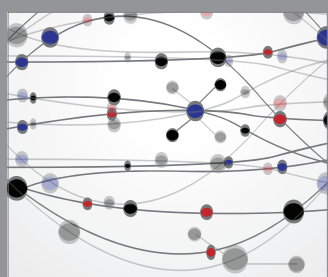

The Scientific World Journal

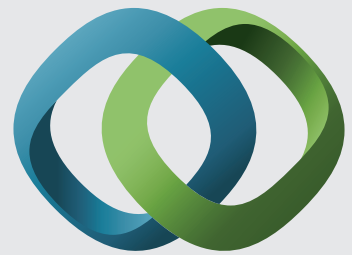

\section{Hindawi}

Submit your manuscripts at

http://www.hindawi.com
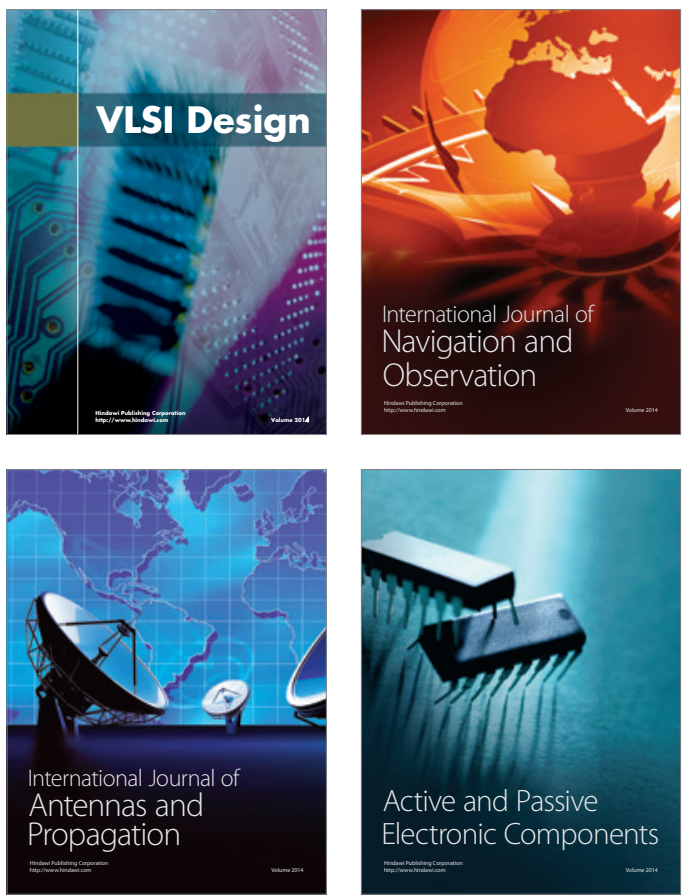
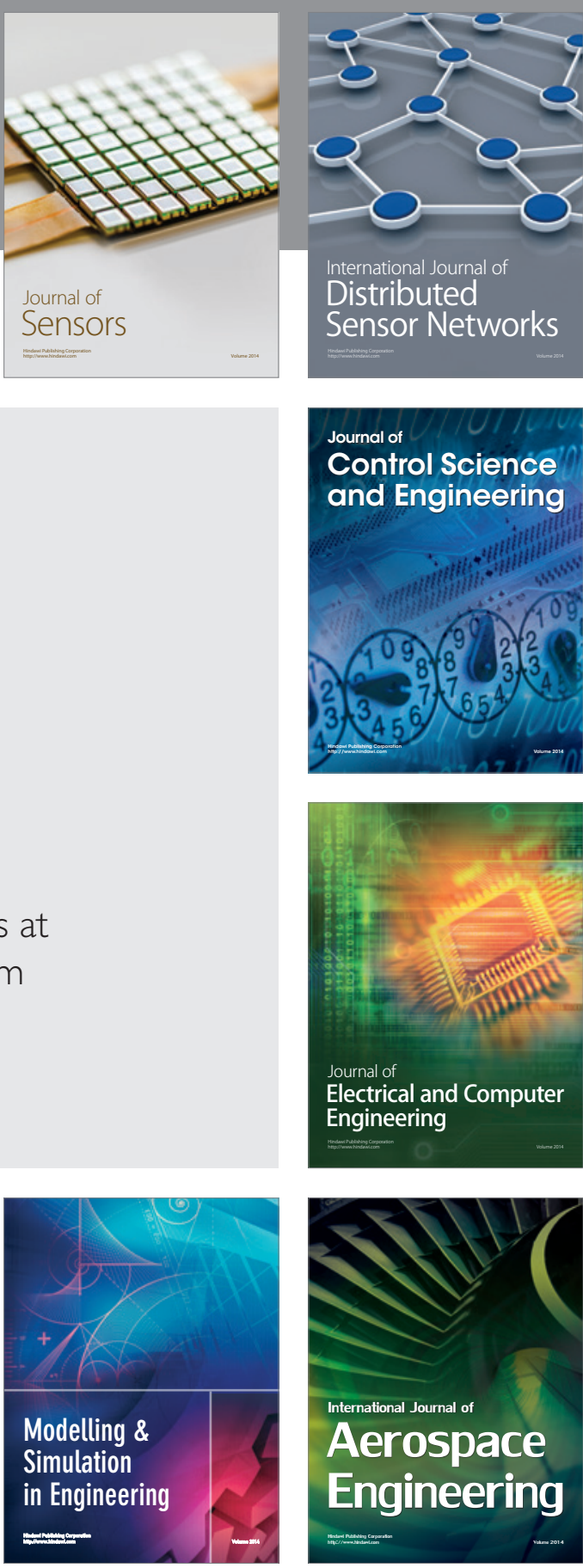

International Journal of

Distributed

Sensor Networks

Journal of

Control Science

and Engineering
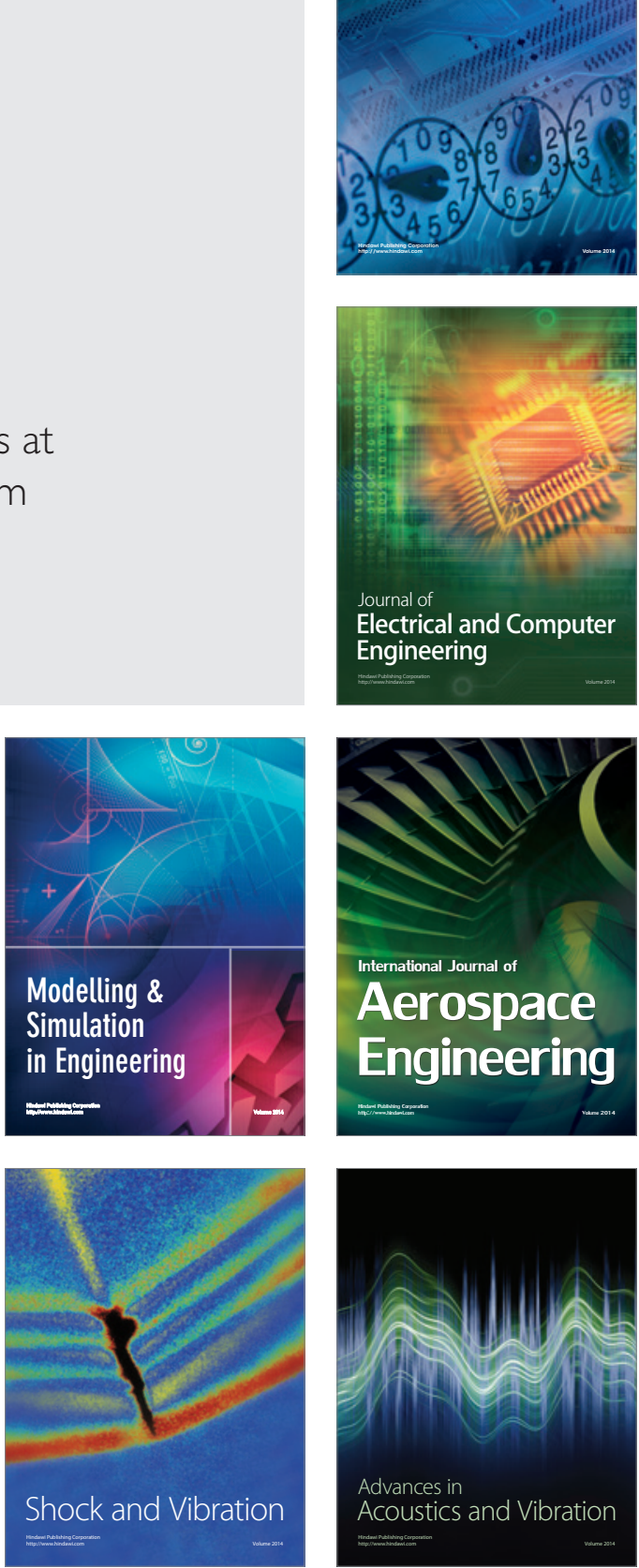